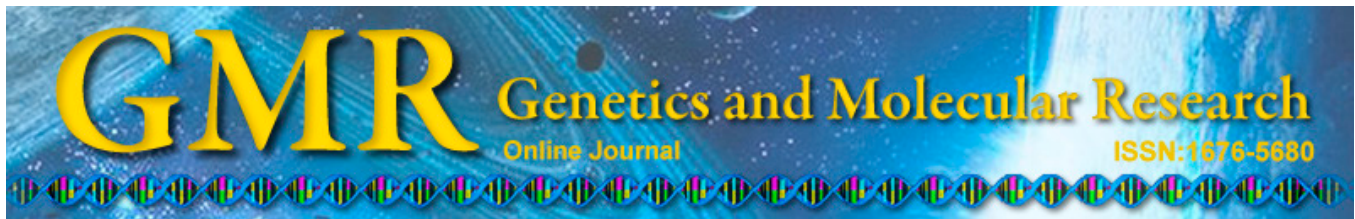

\title{
Detecting circulating tumor cells in patients with advanced non-small cell lung cancer
}

\author{
Y.H. Xu' ${ }^{1 *}$, J. Zhou ${ }^{2 *}$ and X.F. Pan $^{3}$ \\ ${ }^{1}$ Jingzhou Hospital Affiliated to Tongji Medical College of Huazhong \\ University of Science and Technology Jingzhou, Hubei Province, China \\ ${ }^{2}$ Jingzhou Central Hospital Jingzhou, Hubei Province, China \\ ${ }^{3}$ Cancer Center, Jingzhou Central Hospital Jingzhou, Hubei Province, China \\ *These authors contributed equally to study. \\ Corresponding author: X.F. Pan \\ E-mail: panxf2014@126.com
}

Genet. Mol. Res. 14 (3): 10352-10358 (2015)

Received February 28, 2015

Accepted June 23, 2015

Published September 1, 2015

DOI http://dx.doi.org/10.4238/2015.September.1.1

\begin{abstract}
We explored the expression and clinical significance of circulating tumor cells (CTCs) in patients with advanced non-small cell lung cancer (NSCLC). Sixty-six patients with advanced NSCLC at the Oncology Department of Jinzhou Hospital were selected as an observation group between February and December 2013. Healthy volunteers and 20 benign lung disease patients were taken as a control group. Peripheral blood CTCs in the observation and control groups were detected using the CellSearch ${ }^{\circledR}$. CTC detection and analysis system, and the relationship between the expression and clinical effect of CTCs and disease progression was analyzed. Peripheral blood CTCs were observed in 47 of the 66 observation group cases $(71.21 \%)$, but none were found in the control group $(\mathrm{P}<0.05)$. The CTC-positive rate was independent of NSCLC patients' age, gender, smoking habits, histological features, and degree of differentiation $(\mathrm{P}>0.05)$. The CTCpositive rate correlated with pathological staging $(\mathrm{P}<0.05)$. After two
\end{abstract}


courses of chemotherapy, the number of cases with CTCs $\geq 3$ decreased significantly, compared with pre-chemotherapy cases $(\mathrm{P}<0.05)$, and the disease did not progress in 37 cases ( 34 cases with $<3$ CTCs and three cases with $\geq 3$ CTCs). Eight cases displayed disease progression, of which five cases had $<3$ CTCs and three cases had $\geq 3$ CTCs. There was a statistically significant correlation between CTC changes and disease progression $(\mathrm{P}<0.05)$. The CTC-positive rate correlated with the pathological staging and changes in the number of CTCs were associated with chemotherapy efficacy and disease progression.

Key words: Circulating tumor cells; Non-small cell lung cancer; Lung cancer

\section{INTRODUCTION}

Lung cancer is the leading cause of cancer death, and non-small cell lung cancer (NSCLC), which includes squamous cell carcinoma, adenocarcinoma, and large cell carcinoma, accounts for $80-85 \%$ of lung cancer cases (Hanahan and Weinberg, 2011). Adenocarcinoma is the most common form. In recent years, there have been improvements in sensitivity and specificity in the detection of cancer. Studies have shown that circulating tumor cells (CTCs) in the peripheral blood, and the recurrence and distant transfer of breast cancer diagnosis cells are the main cause of death in patients (Nagrath et al., 2007; Zheng and Li et al., 2009). The movement of tumor cells into the peripheral blood is the main pathway of tumor metastasis and the basis of distant metastasis. Currently, CTCs are defined as tumor cells that are released into the peripheral blood from metastases or solid tumors either spontaneously or as a result of medical procedures such as needle biopsy or surgery. Sensitive treatment following detection of CTCs is closely related to prognosis (Xenidis et al., 2009; Lucci et al., 2012). In this study, the expression of CTCs in patients with advanced NSCLC was explored by detecting CTCs in peripheral blood to determine their relationship to chemotherapy efficacy and disease progression.

\section{MATERIAL AND METHODS}

\section{Subjects}

Sixty-six patients with advanced NSCLC at the Oncology Department of Jinzhou Hospital were selected for the observation group between February and December 2013. Thirty-six were male, 30 were female, and they were aged 34-80 years, with a median age of 69 years. All patients enrolled were confirmed with NSCLC by pathological histology or cytology. Patients were hospitalized for the first time, and did not undergo any preanticancer therapy. All patients were expected to survive for more than 3 months. Healthy volunteers and 20 patients with benign lung disease were taken as the control group (aged 33-78 years with a median age of 65 years). The two groups were demographically comparable, with no statistically significant differences in age and gender. All patients signed informed consent agreements. 


\section{Methods}

\section{Instruments and reagents}

The CellSearch ${ }^{\circledR}$ CTC detection and analysis system was provided by Veridex Corp. (a subsidiary of Johnson \& Johnson, New Jersey, USA). It is the only product for the detection of CTCs that is approved by the Food and Drug Administration of the USA and the State Food and Drug Administration of the People's Republic of China. The detection system includes: a CellSave storage tube, the CellSearch kit, the CellTracks automatic response analyzer, a magnetic cell presentation device slot, and the CellTracks automatic response analyzer.

\section{Sample collection and separation}

Specimen were prepared according to the following steps. After rigorous disinfection, cubital vacuum blood was collected using a blood collection needle. After puncture, the initial $2 \mathrm{~mL}$ blood was discarded before $7.5 \mathrm{~mL}$ blood was drawn into the special tube containing ethylenediaminetetraacetic acid anticoagulant (CellSave Preservative Tube). The blood samples were tested within $24 \mathrm{~h}$ of collection.

\section{Sample detection}

Blood $(7.5 \mathrm{~mL})$ was drawn into the tube containing $6 \mathrm{~mL}$ buffer and centrifuged at $800 \mathrm{~g}$ for $10 \mathrm{~min}$. The tube was then placed in the CellSearch system to remove the layered plasma and anticoagulant, and the "epithelial cell-specific reagent", which is a ferrofluid rich in anti-EpCAM antibody, was added. CTCs were immunomagnetically enriched with the ferrofluid. Cytokeratin, CD45, and nuclei were respectively stained with phycoerythrin, allophycocyanin, and 4',6-diamidino-2-phenylindole hydrochloride, and cells were finally suspended in a magnetic cell presentation device slot (Veridex Corp., USA) through repeated immune magnetic separation and fluorescence permeabilizing for analysis using CellSpotter.

\section{Evaluation criteria}

The observation group was treated with cisplatin and gemcitabine to regulate chemotherapy on a 21-day cycle. CTCs were detected before the 1st, 2nd, and 3rd cycles of chemotherapy. The efficacy was defined as complete remission (CR), partial response (PR), stable disease (SD), and progress disease (PD), according to the Solid Tumors Response Evaluation Criteria (Ding et al., 2014).

\section{Statistical analysis}

All statistical analyses were performed using SPSS 13.0. Count data were assessed using the Fisher exact test, paired $\chi^{2}$ tests and $\chi^{2}$ test of rows $\mathrm{x}$ list, where $\mathrm{P}$ values of $\leq 0.05$ were considered to be statistically significant. 


\section{RESULTS}

\section{CTC test results}

CTCs were not found in the patients with benign lung disease or the healthy volunteers in the control group. CTCs were detected in 47 cases of the 66 cases $(71.21 \%)$ in the observation group. There was a statistically significant difference in the CTC-positive rates of the two groups $(\mathrm{P}<0.01)$.

\section{Relationship between CTC test results and clinical characteristics of the patients}

The CTC-positive rate was independent of the NSCLC patients' age, gender, smoking habits, histological features, and degree of differentiation $(\mathrm{P}>0.05)$. The CTC-positive rate correlated with the pathological staging. The CTC-positive rate of the patients with stage IV NSCLC was higher than that of stage IIIB patients; the CTC-positive rate of stage IIIA patients was lowest. The difference was statistically significant $(\mathrm{P}<0.05$, Table 1$)$.

\begin{tabular}{|c|c|c|c|c|c|}
\hline Clinical features & No. of cases & CTC-positive (\%) & CTC-negative (\%) & $\chi^{2}$ & $\mathrm{P}$ values \\
\hline Age & & & & 0.101 & 0.750 \\
\hline$\leq 60$ years old & 19 & $13(68.42)$ & $6(31.58)$ & & \\
\hline$>60$ years & 47 & $34(72.34)$ & $13(27.65)$ & & \\
\hline Gender & & & & 0.062 & 0.803 \\
\hline Male & 36 & $26(72.22)$ & $10(27.77)$ & & \\
\hline Female & 30 & $21(70.00)$ & $7(30.00)$ & & \\
\hline Smoking & & & & 3.767 & 0.052 \\
\hline Yes & 40 & $32(80.00)$ & $8(20.00)$ & & \\
\hline No & 26 & $15(57.69)$ & $11(42.30)$ & & \\
\hline Histological features & & & & 0.002 & 0.967 \\
\hline Adenocarcinoma & 35 & $25(71.43)$ & $10(28.57)$ & & \\
\hline Squamous cell carcinoma & 31 & $22(70.97)$ & $9(29.03)$ & & \\
\hline Differentiation & & & & 0.298 & 0.816 \\
\hline High differentiation & 9 & $6(66.67)$ & $3(33.33)$ & & \\
\hline Moderate differentiation & 36 & $26(72.22)$ & $10(27.78)$ & & \\
\hline Poor differentiation & 21 & $16(76.19)$ & $5(23.81)$ & & \\
\hline Pathological stage & & & & 13.88 & 0.01 \\
\hline IIIA & 7 & $2(28.57)$ & $5(71.43)$ & & \\
\hline IIIB & 23 & $13(56.52)$ & $10(43.48)$ & & \\
\hline IV & 36 & $32(88.89)$ & $4(11.11)$ & & \\
\hline
\end{tabular}

\section{Correlation between CTC and chemotherapy}

A total of 47 patients were enrolled in the observation group, of which there were 15 patients with $\geq 3$ CTCs before chemotherapy (31.91\%). All 47 patients completed the first course of chemotherapy, following which there were 11 cases with $\geq 3$ CTCs $(23.40 \%)$. Fortyfive patients completed the second course of chemotherapy, following which the number of patients with $\geq 3$ CTCs was six $(13.33 \%)$. After the first course of chemotherapy, the number of patients with $\geq 3$ CTCs decreased, but the difference was not statistically significant $\left(\chi^{2}=\right.$ $2.705, \mathrm{P}=0.148)$; after two courses of chemotherapy, the number of patients with $\geq 3$ CTCs decreased significantly and, compared with the pre-chemotherapy group, there was a statistically significant difference $\left(\chi^{2}=4.457, \mathrm{P}=0.047\right.$, Table 2$)$. 
Table 2. Relationship between circulating tumor cell (CTC) test results and chemotherapy.

\begin{tabular}{lccr}
\hline Chemotherapy status & No. of cases & CTCs $<3(\%)$ & CTCs $\geq 3(\%)$ \\
\hline Before chemotherapy & 47 & $32(68.08)$ & $15(31.91)$ \\
After a course of chemotherapy & 47 & $36(76.59)$ & $11(23.40)$ \\
After two courses of chemotherapy & 45 & $39(86.67)$ & $6(13.33)$ \\
\hline
\end{tabular}

\section{Relationship between CTC changes and disease progression}

Before chemotherapy, in the observation group there were 40 patients without disease progression, including 28 cases with $<3$ CTCs and 12 cases with $\geq 3 \mathrm{CTCs}$, and seven patients with disease progression, including four cases with $<3$ CTCs and three cases with $\geq 3$ CTCs. There was no statistically significant difference between CTC changes and disease progression $(\mathrm{P}=0.664)$. After the first cycle of chemotherapy, the disease did not progress in 40 patients, including 32 cases with $<3$ CTCs and eight cases with $\geq 3$ CTCs; disease progressed in seven cases, including four cases of $<3$ CTCs and three cases of $\geq 3$ CTCs. There was no statistically significant difference between CTC changes and disease progression $(\mathrm{P}=0.330)$. After two courses of chemotherapy, the disease did not progress in 37 cases, including 34 cases with $<3$ CTCs and three cases with $\geq 3$ CTCs; eight cases had disease progression, of which there were five cases with $<3$ CTCs and three cases with $\geq 3$ CTCs. There was a statistically significant difference between $\mathrm{CTC}$ changes and disease progression $(\mathrm{P}=0.028$, Table 3$)$.

Table 3. Relationship between change in the number of circulating tumor cells (CTCs) and disease progression.

\begin{tabular}{|c|c|c|c|c|c|c|c|}
\hline & \multicolumn{3}{|c|}{ No disease progression } & \multicolumn{3}{|c|}{ Disease progression } & \multirow[t]{2}{*}{$P$ values } \\
\hline & No. of cases & CTCs $<3$ & $\mathrm{CTCs} \geq 3$ & No. of cases & CTCs $<3$ & $\mathrm{CTCs} \geq 3$ & \\
\hline Before chemotherapy & 40 & 28 & 12 & 7 & 4 & 3 & 0.664 \\
\hline After a course of chemotherapy & 40 & 32 & 8 & 7 & 4 & 3 & 0.330 \\
\hline After two courses of chemotherapy & 37 & 34 & 3 & 8 & 5 & 3 & 0.028 \\
\hline
\end{tabular}

\section{DISCUSSION}

Bone puncture and venous sampling are the two main methods for detection of transferred cancer cells in blood. A large number of studies have shown that disseminated tumor cells can be detected in most of cancer patients' bone marrow. The bone puncture method is time-consuming and causes discomfort to the patient, which is difficult for the patient to accept, and results in poor compliance. The detection of circulating tumor cells in peripheral blood is time-saving, safe, and repeatable (Allan and Keeney, 2010). In recent years, with the improvements in sensitivity and specificity in CTC detection technology, more attention has been paid to the relationship between the number of peripheral blood CTCs and prognosis and chemotherapy efficacy.

Currently, CTC detection technology usually enriches and separates CTCs first, and then identifies and confirms the result. Different enrichment and identification methods are combined for detection technology, but a variety of test results show significant differences. The detection techniques lack a standard and automatic program, and require tedious sample preparation procedures. Different detection methods lead to different sensitivity and specificity. Therefore, detection of CTCs is not applied in routine clinical tests. 
The CellSearch circulating tumor cell detection and analysis system is an advanced diagnostic and research platform able to analyze the micro-cell by using immune nanometer magnetic particle patented technology. The system is able to trace blood cells for accurate analysis, including, but not limited to, CTCs derived from epithelial and circulating endothelial cells, and it has good reproducibility. It belongs to a semi-automatic detection system that automatically uptakes and detects CTCs by immunocytochemistry. It facilitates the detection of peripheral blood CTCs in a clinical setting.

In this study, CTCs in the peripheral blood of 66 patients with NSCLC were detected using the CellSearch system. CTCs were detected in 47 cases (71.21\%), and the detection rate was higher than in similar studies using other detection methods (Wu et al., 2009; Zhou et al., 2010). Furthermore, none of the 20 patients in the control group were found to be CTCpositive, indicating that the CTC detection system has high sensitivity and specificity. The relationships between the CTC-positive rate and the clinical characteristics of the patients were noted. We found that CTC-positive rate was independent of the NSCLC patient's age, gender, smoking habits, histological features, and degree of differentiation $(\mathrm{P}>0.05)$. The CTC-positive rate correlated with the pathological staging. The CTC-positive rate of the patients with stage IV NSCLC was higher than those with stage IIIB; the CTC-positive rate of the stage IIIA patients was the lowest. The difference was statistically significant $(\mathrm{P}<0.05)$. This result is consistent with the reported studies (Li et al., 2013).

The correlation between CTCs and chemotherapy was also explored. It showed that there were 15 patients with $\geq 3$ CTCs before chemotherapy (39.91\%). After the first course of chemotherapy, there were 11 cases with $\geq 3$ CTCs $(23.40 \%)$, but the difference was not statistically significant $(\mathrm{P}>0.05)$. After the second course, the number of patients with $\geq 3 \mathrm{CTCs}$ was six $(13.33 \%)$. There was a statistically significant difference in the number of patients with $\geq 3$ CTCs compared with pre-chemotherapy patients $(\mathrm{P}<0.05)$. Studies have shown that CTCs in the peripheral blood of patients can be reduced to a certain extent with chemotherapy; CTC detection can indicate the effect of chemotherapy to some degree.

Hirose et al. (2012) carried out the CTC detection in patients with metastatic NSCLC before and after chemotherapy. The transferred rate of progression after chemotherapy showed that the progression of CTC-positive patients $(66.7 \%)$ was higher than that of CTC-negative patients $(23.8 \%)$. However, the study did not mention the impact of CTC number on the rate of disease progression in CTC-positive patients. The relationship between the number of CTCs in peripheral blood and disease progression was analyzed in this study. Our study showed that in CTC-positive patients, before chemotherapy and at the end of the first course, there was no significant statistical difference between the number of CTCs in disease progression and progression-free patients $(\mathrm{P}>0.05)$. After the second course of chemotherapy, the number of CTCs in disease progression and progression-free patients showed a significant statistical difference $(\mathrm{P}<0.05)$. The study further revealed the indicative role of dynamic changes of CTCs in chemotherapy efficacy and disease prognosis.

In summary, with the continuous development of medical devices, CTC detection as an emerging technology represents an improvement in specificity, sensitivity, and clinical convenience. Its role in prognosis and determining chemotherapy efficacy in cancer therapy has also attracted the attention of a growing number of researchers. As it continues to develop, CTC detection has great potential within the field of lung cancer therapy, and may provide new guidance in the choice of personalized drug treatment. 


\section{Conflicts of interest}

The authors declare no conflict of interest.

\section{REFERENCES}

Allan AL and Keeney M (2010). Circulating tumor cell analysis: technical and statistical considerations for application to the clinic. J. Oncol. 2010: 426218.

Ding Q, Cheng X, Yang L, Zhang Q, et al. (2014). PET/CT evaluation of response to chemotherapy in non-small cell lung cancer: PET response criteria in solid tumors (PERCIST) versus response evaluation criteria in solid tumors (RECIST). J. Thorac. Dis. 6: 677-83.

Hanahan D and Weinberg RA (2011). Hallmarks of cancer: the next generation. Cell 144: 646-674.

Hirose T, Murata Y, Oki Y, Sugiyama T, et al. (2012). Relationship of circulating tumor cells to the effectiveness of cytotoxic chemotherapy in patients with metastatic non-small-cell lung cancer. Oncol. Res. 20: 131-137.

Li J, Jiang B, Wan P, Li X, et al. (2013). The clinical significance of late detection of circulating tumor cells in patients with non-small cell lung cancer. Chin. Gen. Pract. 16: 3202-3207.

Lucci A, Hall CS, Lodhi AK, Bhattacharyya A et al. (2012). Circulating tumour cells in non-metastatic breast cancer: a prospective study. Lancet Oncol. 13: 688-695.

Nagrath S, Sequist LV, Maheswaran S, Bell DW et al. (2007). Isolation of rare circulating tumour cells in cancer patients by microchip technology. Nature 450: 1235-1239.

Wu C, Hao H, Li L, Zhou X, et al. (2009). Preliminary investigation of the clinical significance of detecting circulating tumor cells enriched from lung cancer patients. J. Thorac. Oncol. 4: 30-36.

Xenidis N, Ignatiadis M, Apostolaki S, Perraki M, et al. (2009). Cytokeratin-19 mRNA-positive circulating tumor cells after adjuvant chemotherapy in patients with early breast cancer. J. Clin. Oncol. 27: 2177-2184.

Zheng J and Li L (2009). Research status of circulating tumor cells in breast cancer. J. Mod. Oncol. 17: 1158-1162.

Zhou XJ, Li LY, Hao HJ, Sun L, et al. (2010). Predictive and prognostic value of tumor cells in the peripheral circulation study in patients with advanced lung cancer. Oncol. Progr. 5: 484-490. 